\title{
An Overview of Variable Aperture Mechanisms in Attempt to Control Temperature Inside Solar Cavity Receivers
}

\section{Conference Paper · March 2018}

CITATIONS

0

5 authors, including:

\section{Cédric Ophoff}

University of Leuven

5 PUBLICATIONS 11 CITATIONS

SEE PROFILE

\section{Nesrin Ozalp}

University of Minnesota Duluth

85 PUBLICATIONS 699 CITATIONS

SEE PROFILE
READS

35

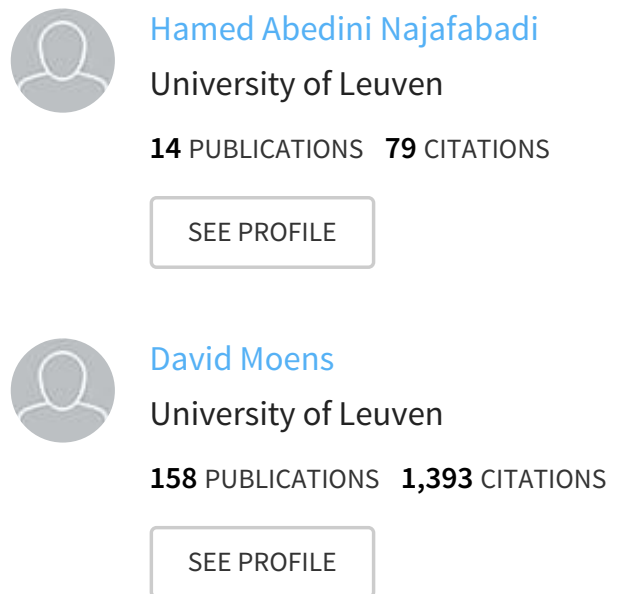

Some of the authors of this publication are also working on these related projects:

Project

Project
Development and validation of high-definition numerical methods for mechanical design under manufacturing process variation View project

KU Leuven Noise \& Vibration Research Group View project 


\title{
AN OVERVIEW OF VARIABLE APERTURE MECHANISMS IN ATTEMPT TO CONTROL TEMPERATURE INSIDE SOLAR CAVITY RECEIVERS
}

\author{
C. Ophoff ${ }^{1}$, H. Abedini Najafabadi² ${ }^{2}$ J. Bogaerts ${ }^{1}$, N. Ozalp ${ }^{3 *}$, D. Moens ${ }^{1}$ \\ ${ }^{1}$ Mechanical Engineering Department, KU Leuven, 3001 Leuven, Belgium \\ ${ }^{2}$ School of Chemical Engineering, Iran University of Science and Technology, Tehran, Iran \\ ${ }^{3}$ Mechanical and Industrial Engineering Department, University of Minnesota Duluth, 55812 Duluth, \\ Minnesota, USA
}

\begin{abstract}
In solar thermal research, there is a lot of attention has been paid on optimal design of solar reactors and cavity receivers in order to overcome inherent technical challenges. While many of these studies focus on the geometry and flow optimization of the reactor/receiver, few have focused on control of light entry into the cavity via auxiliary embedded mechanisms. Because the natural fluctuation of solar radiation affecting the thermal behavior of these reactor/receivers during daytime, it is important to address this challenge. In order to cope with transient nature of solar energy, several modular devices have been conceived. They are designed based upon concepts featuring control of set of blades or interchanging apertures to adjust light entry. These techniques yield promising performance on maintaining the desired reactor temperature and solar to fuel efficiencies. Towards that effort, an extensive fundamental background in designing, manufacturing, and testing of such mechanisms has been accumulated by our research group. This paper provides an overview of three successful mechanical apertures developed by our group to regulate flux entry into solar thermal receivers. The overview covers methodological aspects commonly encountered during the design process. A thorough comparison of performance records of these mechanisms are given per their actuation methods. Effect of each aperture mechanism and their individual change of cross-sectional shape is being evaluated through extensive optical simulation. The simulation results give insights on their respective optical performance by quantifying their radiative energy gain and losses. The paper also presents a numerical method coupling optical model to a thermodynamic analysis yielding accurate estimation of reactor temperature per experimental validation. Finally, numerical simulation showed that closed loop control of aperture size can efficiently regulate the temperature throughout a sunny day. Results of this research highlight the advantage of adopting variable apertures in solar cavity receivers.
\end{abstract}

KEYWORDS: Solar reactor/receiver; Aperture; Mechanical design; Optical analysis; Temperature control

\section{INTRODUCTION}

The sun is an inexhaustible resource of renewable energy. This energy can be used to supply high process heat that is necessary to operate thermochemical process, for production of various solar fuels and commodities. Solar radiation can be concentrated by the use of mirrors tracking the motion of the sun. This concentrated beam of solar energy is directed towards a cavity called the "solar reactor" or "solar receiver". The intensity of solar radiation, however, is not constant. Meteorological conditions, seasons, and position of the sun change the incoming flux rates continuously. This makes it difficult to maintain a constant temperature in the reactor, resulting in lower process efficiency. To make these fluctuations less disturbing, the solar reactor is equipped with an iris mechanism that controls the amount of concentrated sunlight entry into the reactor. Variable apertures are being used in different applications and domains. Examples of these 
applications are cameras in photography, flow control in chemical processes (control valves), used as a heat shield for furnace windows, and many other applications requiring periodic or continuously variable flow control of material, gas, or fluid. However, use of variable aperture concept in solar reactor technology is new and pioneered by our research group.
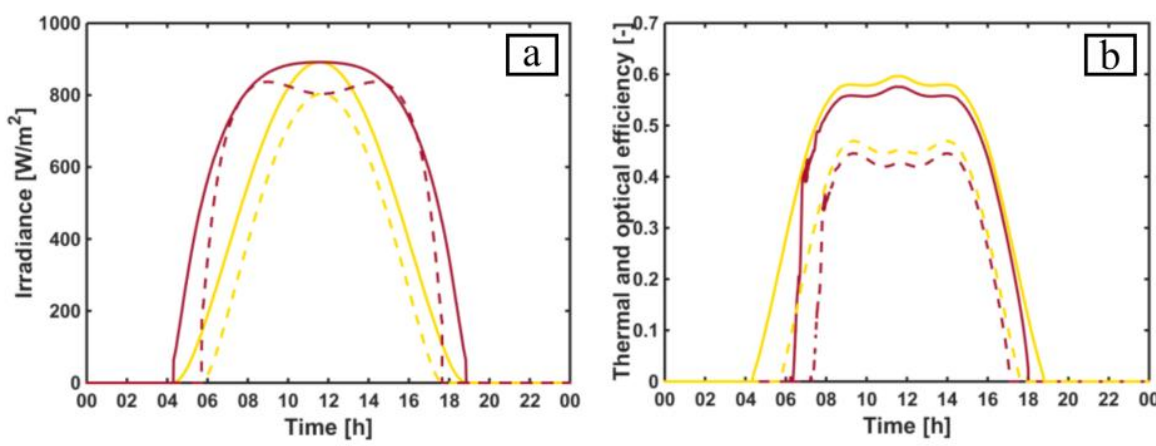

Fig. 1 (a) Direct Horizontal Irradiance (DHI, yellow) versus Direct Normal Irradiance (DNI, red), (b) Solar field thermal (red) versus optical (yellow) efficiency [Edited from Ref. 1, Figures 2-3, p. 070014-6]

The Earth receives solar energy at a rate of $1.7 \times 10^{17} \mathrm{~W}$ which is in the order of $100-800 \mathrm{~W} / \mathrm{m}^{2}$ on the surface depending on the season and region. By concentration, it is possible to achieve enough energy for the production of electricity, fuels and commodities. However, varying position of the sun leads to fluctuating levels of insulation which reduces the efficiency of solar reactor. Fig. 1 shows a Gaussian distribution of the insolation from sunrise to sunset for two different days of the year, distinguished by the line style. From 9 am until $15 \mathrm{pm}$ the efficiencies reach their maximum of approximately $45 \%$ and $55 \%$ for both days respectively [1]. Use of an adaptable aperture makes it possible to reach a more constant thermal output that would result in higher overall efficiencies. Therefore, it is important to design a mechanism that can improve the efficiencies of solar radiation driven energy conversion systems.

To address the problem of low production efficiency because of fluctuating solar energy, this paper presents an overview of variable aperture mechanisms that are used in conjunction with solar cavity receivers. The present study includes a novel concept for a variable-size aperture inspired by an iris flower, where the petals of the flower close in the dark and open when exposed to light.

\section{APERTURE CONCEPTS IN LITERATURE}

Apertures designed by several authors found in literature, such as those created by Ref. [2], Ref. [3] and Ref. [4], served as an inspiration for developing one of the mechanisms presented in this paper. The designs that are specifically examined here were done by Ref. [3], Ref. [5], Ref. [6], and Ref. [7]. For example, Ref. [5] developed a mechanism for flow control. In their design, a drive ring is provided with a rack gear, actuated by a gear mounted on a shaft. The curved blades are pivotally mounted on the mounting ring which provides sliding holes for it. Offset pins on the blades are located in grooves of the drive ring. The blades are in an overlapping position, so the mechanism does not block during actuation. By rotating the drive ring which is actuated by a drive gear; movement of the offset pins in grooves allow the blades to move and thereby vary the area of the aperture. The same actuator principle is used by Ref. [3] and Ref. [6]. These mechanisms are called pin-slot mechanisms.

In another example, Ref. [7] developed a variable aperture concept for a photographic camera to provide proper exposure of the film under different light conditions. When a solenoid coil is energized by a current, a magnetic field will be generated with an amplitude proportional with the amplitude of the current flowing. The solenoid coils are mounted on coil forms which are attached to arm portions of the diaphragm members. A shielding is used to concentrate the magnetic field of the permanent magnet. These elements are used to form a solenoid actuator which responds to electric current. Spindle members are used with bearings, so the blades can rotate and the aperture area varies. In this figure, the aperture area is an oval, and a spur gear is 
used with members having gear teeth. The aperture geometry can be easily made circular using more overlapping blades.

A design by Ref. [8] was developed as an electromagnetic liquid iris. An electric coil is wrapped around the cylinder cell. When current is applied, the ferro-fluid is actuated and pulled towards the center point. Initially, the ferro-fluid is placed in a sidewall that is given a special hydrophilic treatment so it gets attracted to the inner wall. In neutral condition, the aperture is open. When current is applied, the aperture closes. It should be noted that none of these designs are made for high temperature processes. The following sections provide a discussion about the design methodology and capabilities of the proposed variable aperture mechanisms used in conjunction with solar cavity receivers.

\section{OVERVIEW OF DESIGN FEATURES}

\subsection{Aperture Concepts}

Illustrated in Fig. 2 are three modular devices, proposed and used as variable aperture for a solar cavity receiver. Each design features a different actuation method as will be discussed in further detail below. The iris mechanism (Fig. 2a) comprises a set of eight blades set in motion through a pin-slot actuation disc. A stepper motor delivers the required torque via chain transmission to the disc. The iris mechanism had previously been introduced and described in Ref. 3, Ref. 9 and Ref. 10. The rotary aperture, depicted in Fig. $2 \mathrm{~b}$, is composed of a $53 \mathrm{~cm}$ diameter disc rotating 360 degrees. Thus, twelve predefined aperture sizes can interchangeably be switched. The $3 \mathrm{~mm}$ thick disc is aligned flush with the reactor front flange such that reradiative losses through the aperture are significantly diminished. The rotary aperture and iris mechanism had previously been benchmarked against each other [10]. Results of this study showed that overall higher reactor temperatures were achieved with the rotary aperture for equivalent aperture sizes.
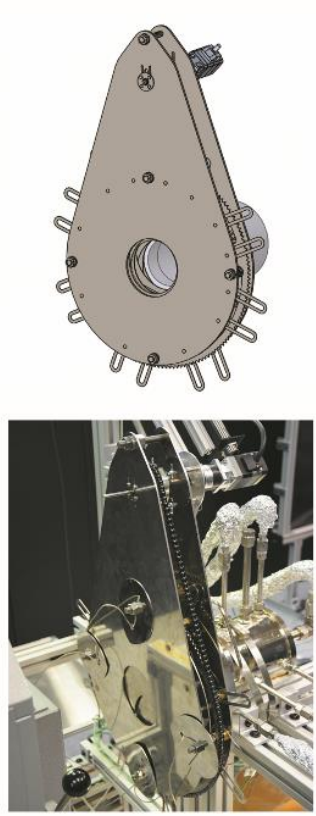

(a)
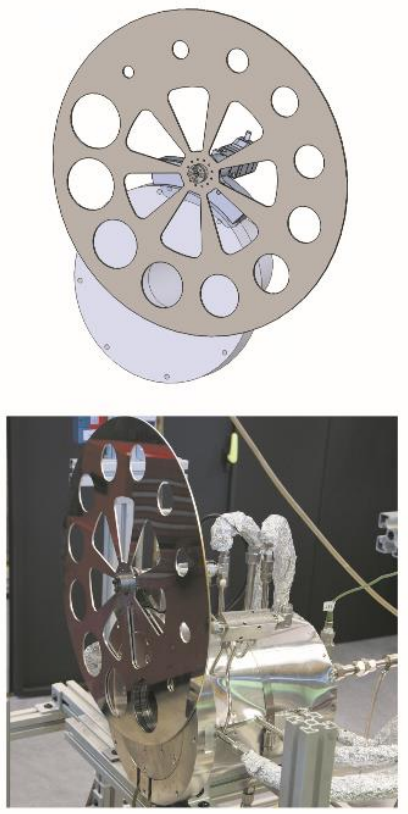

(b)
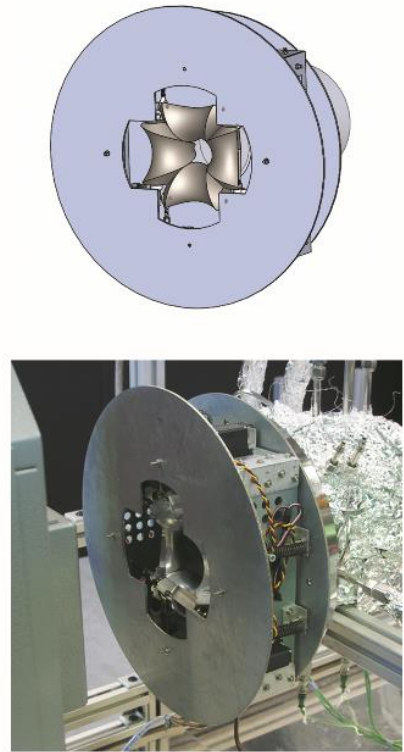

(c)

Fig. 2 Overview of developed aperture concepts mounted on solar reactor: (a) iris mechanism, (b) rotary aperture, and (c) flower mechanism

Inspired by natural mechanisms, the third aperture mechanism was conceived (Fig. 2c) accordingly. Unlike the (multi-)planar motion in the first two designs, the latter mechanism proposes a combined rotational mobility of four solid blades. Revolution of the blades around their longitudinal axis yield a close to circular aperture shape. The 4-blade assembly is mounted in a frame and each blade is individually actuated by a DC- 
servomotor. Combined use of a telescopic universal joint and spring plunger compensate for alignment errors due to thermal expansion.

All three mechanisms are able to enlarge and shrink their aperture in a range of 20 to $90 \mathrm{~mm}$ of diameter. Other key technical design constraints all three mechanisms had to comply with were (1) high temperature resistant material properties for the parts exposed to the high intensity light beam, (2) circularity of the projected aperture shape, and (3) minimized thickness of the mechanism assembly in the optical axis direction. Table 1 presents a comparative matrix of the mechanism's design characteristics.

Table 1 Design characteristics of the variable aperture mechanisms

\begin{tabular}{l|c|c|c}
\hline & Iris mechanism & Rotary aperture & Flower mechanism \\
\hline $\begin{array}{l}\text { No. of blades } \\
\text { Actuation and }\end{array}$ & 8 & N/A & 4 \\
transmission & Pin-slot + Chain & Direct rotation & Direct rotation \\
Mechanism thickness & $60 \mathrm{~mm}$ & $3 \mathrm{~mm}$ & $90 \mathrm{~mm}$ \\
Approximate weight & $10 \mathrm{~kg}$ & $5.5 \mathrm{~kg}$ & $7 \mathrm{~kg}$ \\
Order of manufacturability & 2 & $1(\mathrm{easy})$ & $3($ complex $)$ \\
Aperture diameter range & $0-100 \mathrm{~mm}$ & $20-90 \mathrm{~mm}$ & $0-100 \mathrm{~mm}$ \\
\hline
\end{tabular}

\subsection{Blade Design}

Blade design is the most critical and important part of the overall mechanism. Creativity of a blade defines the uniqueness of the design and makes it stand with its novelty among all other designs. An important constraint associated with this aspect of the design is the circular area variation. The circularity is primarily defined by the blade geometry. Both the iris and the flower mechanism have adopted the use of blades. Their geometry, motion and projected aperture area are demonstrated in Fig. 3.

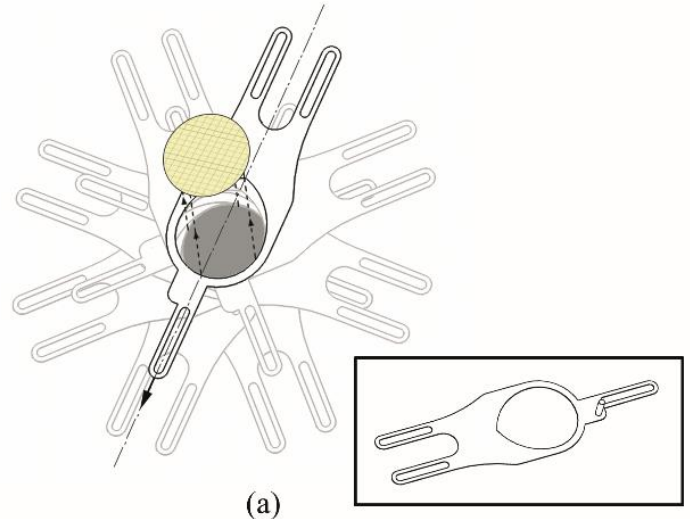

(a)

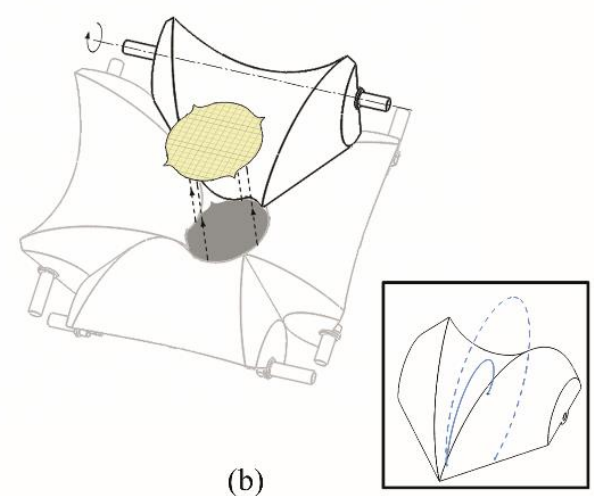

(b)

Fig. 3 Blade geometry and their respective motion for (a) the iris mechanism, and (b) the flower mechanism

Similar to the geometric singularity of the iris blade, which had been previously reported in Ref. 3, the blade concept in the flower mechanism is unique given the variable arch of a circle that follows a curved path and a guided curve. The guided curve was first inserted as a spline sketch in the CAD model, afterwards it was replaced by a mathematical equation. The curved path is equal to three quadrants of a circle which is illustrated by the dashed blue line in Fig. $3 \mathrm{~b}$ and is placed along the wall of a cylinder with radius of $50 \mathrm{~mm}$ and a height of $100 \mathrm{~mm}$. The guided curve guides the arch sketch like a circle with the center of the circle located at the beginning of the curved path. Removing this variable arch, and making upper and lower surfaces of the cylinder conical shaped with an angle of $90^{\circ}$ completed the initial stage of the blade design compilation as shown in Fig. 3b. Initially a rotation of $270^{\circ}$ of each blade was required to completely 
open/close the aperture, which would interfere with the incoming bundle of rays. After scaling the preliminary configuration this rotation was reduced to $180^{\circ}$ and, thus, resolving the issue.

\subsection{Evolution of the Aperture Area}

The most important aspect of the aperture mechanism to investigate is the resulting opening or area which is exclusively depending on the position of the blades. Aforementioned constraints listed circularity as a primary requirement. This follows from the heat flux characterization of the $7 \mathrm{~kW}$ solar simulator, used in the experimental setup, which showed strong resemblance of the obtained flux map with a Gaussian distribution [10, 11]. Similarly, this approximation of the acquired flux density map also applies at larger scale solar concentrator facilities [12-14]. Solar furnaces, for example, generate flux maps with Gaussian-like profile. The deviation in latter case is larger than the solar simulator due to the parabolic dish opposed to an ellipsoidal reflector. The axisymmetry of the flux at the aperture plane of the reactor should therefore be extended to the mechanism geometry.

Evolution of the aperture at different radial positions of the motor shafts is depicted in Fig. 4. It is evident that the aperture holes in the rotary disc are perfectly circular. One of the inherent drawbacks of this variable aperture, however, is the inability to accommodate continuous light entry when interchanging between two subsequent sizes. In addition, implementation of adaptive control on this mechanism to maintain a stable reactor temperature level would often require intermittent switching between two adjacent aperture sizes. In contrast, the blade mechanisms yield an almost infinite range of apertures in the range of 0 to $100 \mathrm{~mm}$ of diameter. It is seen in Fig. 4 that both devices approach a quasi-circular aperture. While the aperture of the iris mechanism transitions between circular and polygonal shapes, the flower mechanism maintains a more constant geometric shape. The circumference of the latter is formed by four circular quadrants with an outward facing groove at the interface of every two blades. This cross-sectional area is clearly shown in Fig. 3b.

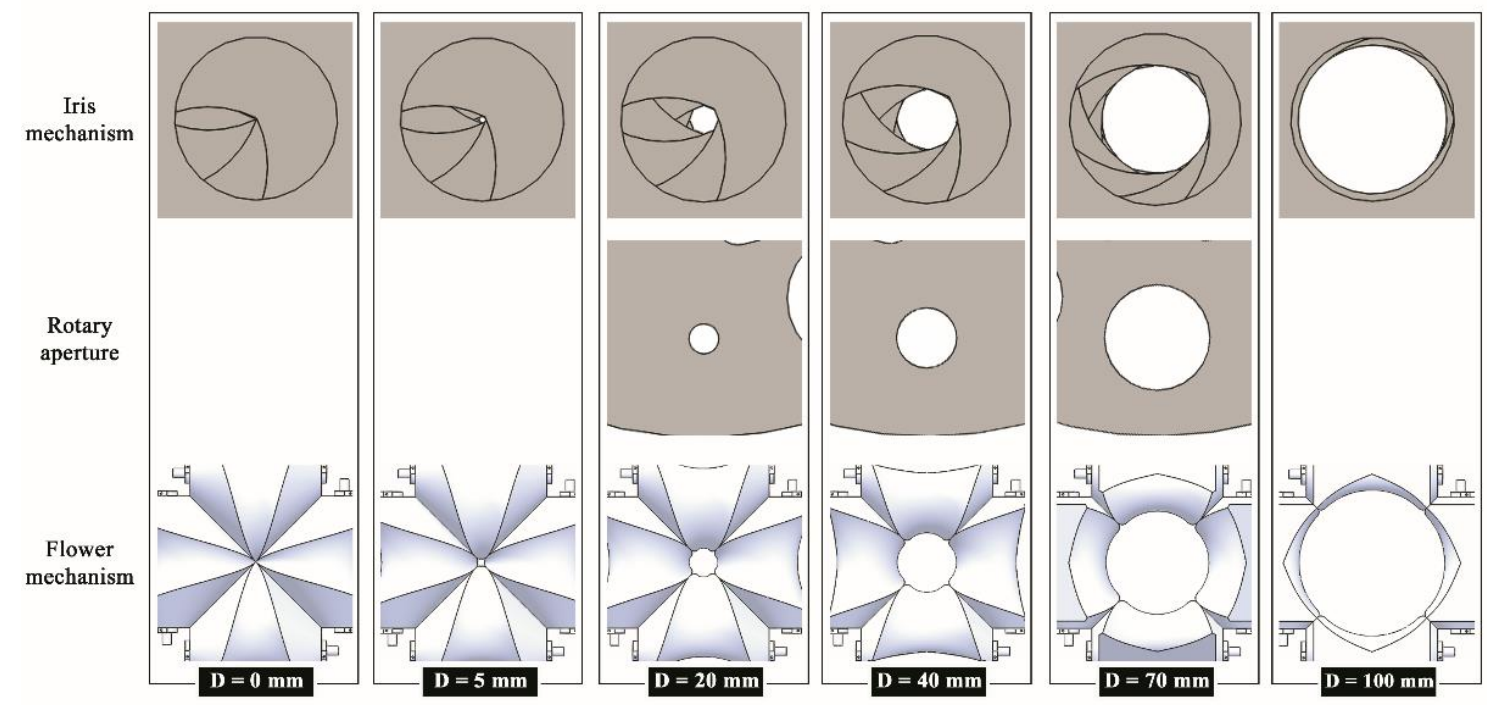

Fig. 4 Gradual change of aperture area and shape for the three mechanisms at six aperture sizes

\section{OPTICAL PERFORMANCE ANALYSIS}

Flux mapping of the solar simulator is the most accurate method to quantify radiative power input of the solar receiver-reactor. Calorimetrically correlated grayscale images captured by an industrial grade camera provide key parameters such as peak flux, total incident power and overall flux density distribution with insignificant errors. This type of flux characterization facilitates repeatability for various power levels of the simulator. However, the mapping method becomes cumbersome and physically impractical when determining flux intercepted by the presented aperture mechanisms. In order to have an accurate estimation 
of the generated flux densities, implementation of an optical model is required. In this section the optical performance of all three mechanisms is assessed.

The optical model was obtained from fitting numerical and experimental flux data [10]. TracePro ${ }^{\circledR}$ optical software, which adopts the Monte Carlo ray tracing algorithm, was used for this optical analysis [15]. Identical surface and material properties were applied to the mechanisms in order to provide consistent and valid results. All surfaces are assumed gray and opaque. An absorptivity-to-reflectivity ratio of 0.67 was assigned to the mechanisms while the inner cavity of the receiver was modelled with a ratio of 4 , which corresponds to their respective material properties [3]. Simulation runs were conducted for the highest achievable power level of the solar simulator, which correlates to $4 \mathrm{~kW}$ of radiative power emitted by the xenon arc. This yields approximately $900 \mathrm{~W}$ at the focal plane, experimentally measured for a target size of $120 \mathrm{x} 120 \mathrm{~mm}^{2}$. Simulation results showed a converged value of $1478 \mathrm{~W}$ of incident flux at the focal plane.

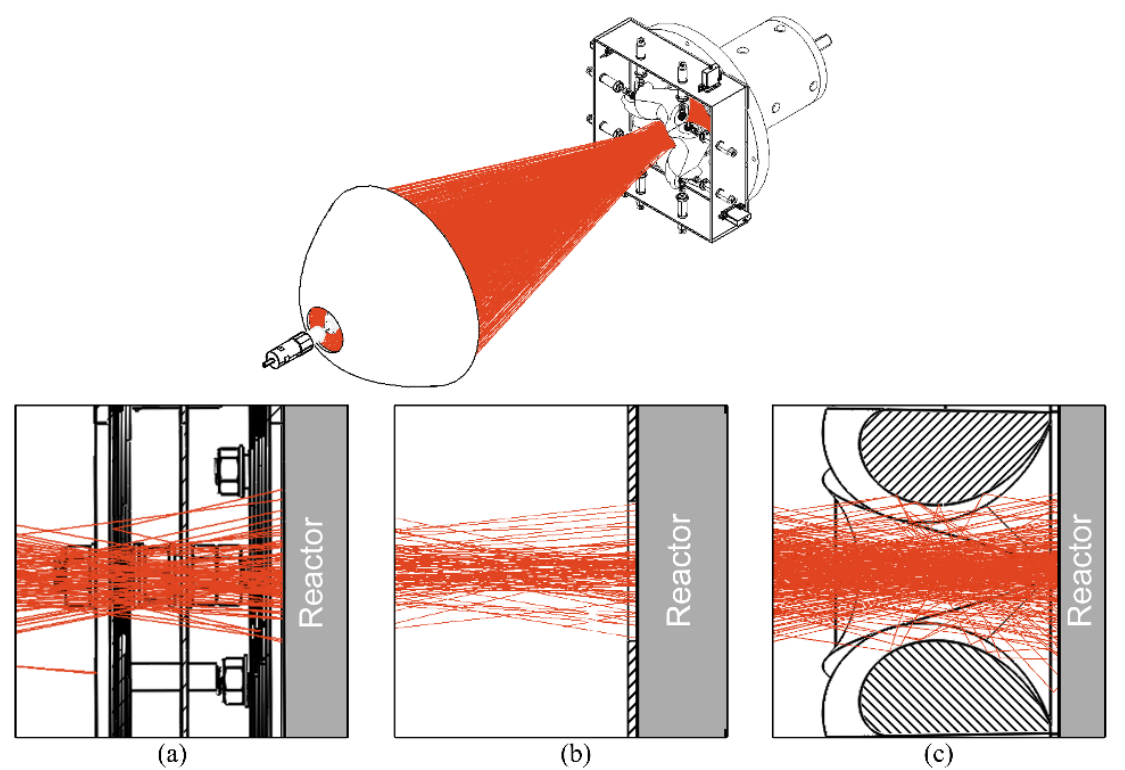

Fig. 5 Optical simulations for three variable aperture mechanisms for $\mathrm{D}=50 \mathrm{~mm}$ : cross-section of (a) iris mechanism, (b) rotary aperture, and (c) flower mechanism

Fig. 5 illustrates the setup used in the simulation model of the flower iris. Fig. 5a-c show the cross-sectional views of the respective mechanisms with $0.01 \%$ of the $2 \mathrm{M}$ generated rays. The equivalent aperture size is compared for each of these in their available range. Fig. 5 shows the simulation runs for a diameter of 50 $\mathrm{mm}$. Simulation model was validated according to the measured flux maps on a $80 \mathrm{x} 80 \mathrm{~mm}^{2}$ target with absolute deviation of $7.6 \mathrm{~W} / \mathrm{m}^{2}$ for peak flux and $0.375 \mathrm{~mm}$ for the half width, as shown in Fig. 6a [10]. Cross-section along the major (x) axis of the resulting flux maps on a $120 \mathrm{x} 120 \mathrm{~mm}^{2}$ target placed behind the aperture are shown in Fig. 6b-d. The peak flux densities observed in the Gaussian-like distributions are respectively $3092 \mathrm{~kW} / \mathrm{m}^{2}$ (iris mechanism), $3092 \mathrm{~kW} / \mathrm{m}^{2}$ (rotary aperture) and $3225 \mathrm{~kW} / \mathrm{m}^{2}$ (flower mechanism). The total fluxes intercepted by the given aperture size were computed and found to be $657 \mathrm{~W}$, $622 \mathrm{~W}$ and $752 \mathrm{~W}$. It is apparent from Fig. 5 that the blades and geometrical parameters of the apertures play a significant role on the optical path of the rays. The planar and multi-planar configurations of, respectively, the rotary aperture and iris mechanism, either block or transmit the light bundles. The blades of the flower mechanism, on the other hand, reflect a fraction of the incoming rays intersecting the mechanism beyond the $50 \mathrm{~mm}$ diameter. This translates into approximately $10 \%$ more rays intercepted by the flower mechanism opposed to the planar mechanisms, and 15 to $20 \%$ more flux is incident on the target. The effect of the blade is also reflected in the tails of the bivariate distributions in Fig. 6. The asymmetry in Fig. $\mathbf{6 b}$ is due to the combined multi-planar and shifted positioning of the iris blades. Unlike the iris and rotary aperture, the enhanced reflectivity of the flower blades spreads the rays on the target and smooths out the tails. In addition, peak flux in Fig. 6 d is $4 \%$ higher than the planar mechanisms. 


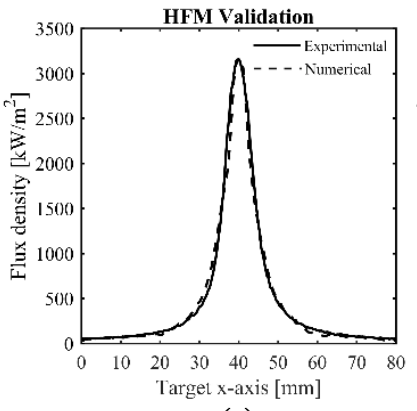

(a)

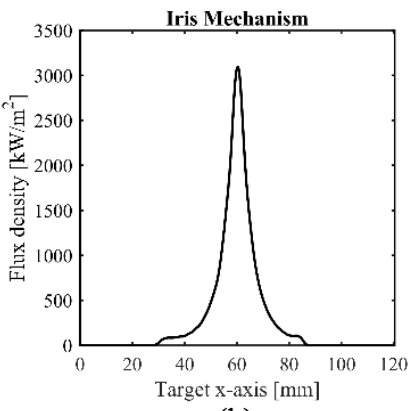

(b)

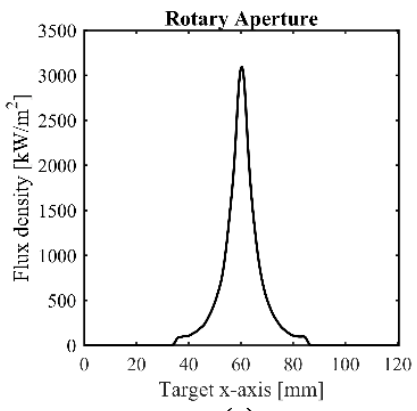

(c)

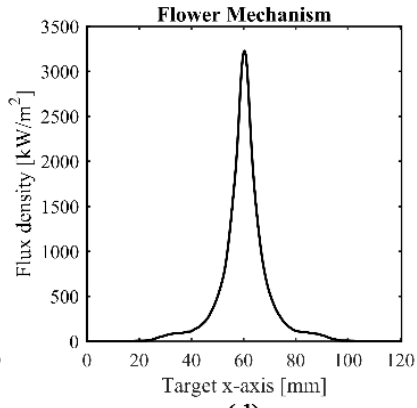

(d)

Fig. 6 (a) Validation of the experimentally measured flux and numerical simulation, and simulated flux intercepted by (b) iris mechanism, (c) rotary aperture, and (d) flower mechanism, respectively, for aperture size of $\mathrm{D}=50 \mathrm{~mm}$

The simulation runs described above provide a good representation of the spatially distributed flux as input to the reactor. A second set of simulation runs was performed and provided insights into the flux distribution incident on the inner cavity walls of the reactor. It is utmost important to achieve and maintain uniform flux distribution, as it inherently yields uniform temperature throughout the reactor which then enhances the solar-to-fuel efficiency. Fig. 7 visualizes the irradiation of the cavity walls. Distribution of the flux density on the inner shell was examined for each mechanism and compared for three aperture sizes. The distribution was plotted against the dimensionless distance in longitudinal and radial direction, respectively. All values were radially averaged. It is clear from the results in Fig. 7 that smaller aperture sizes have a slightly more significant impact on the flux distribution.
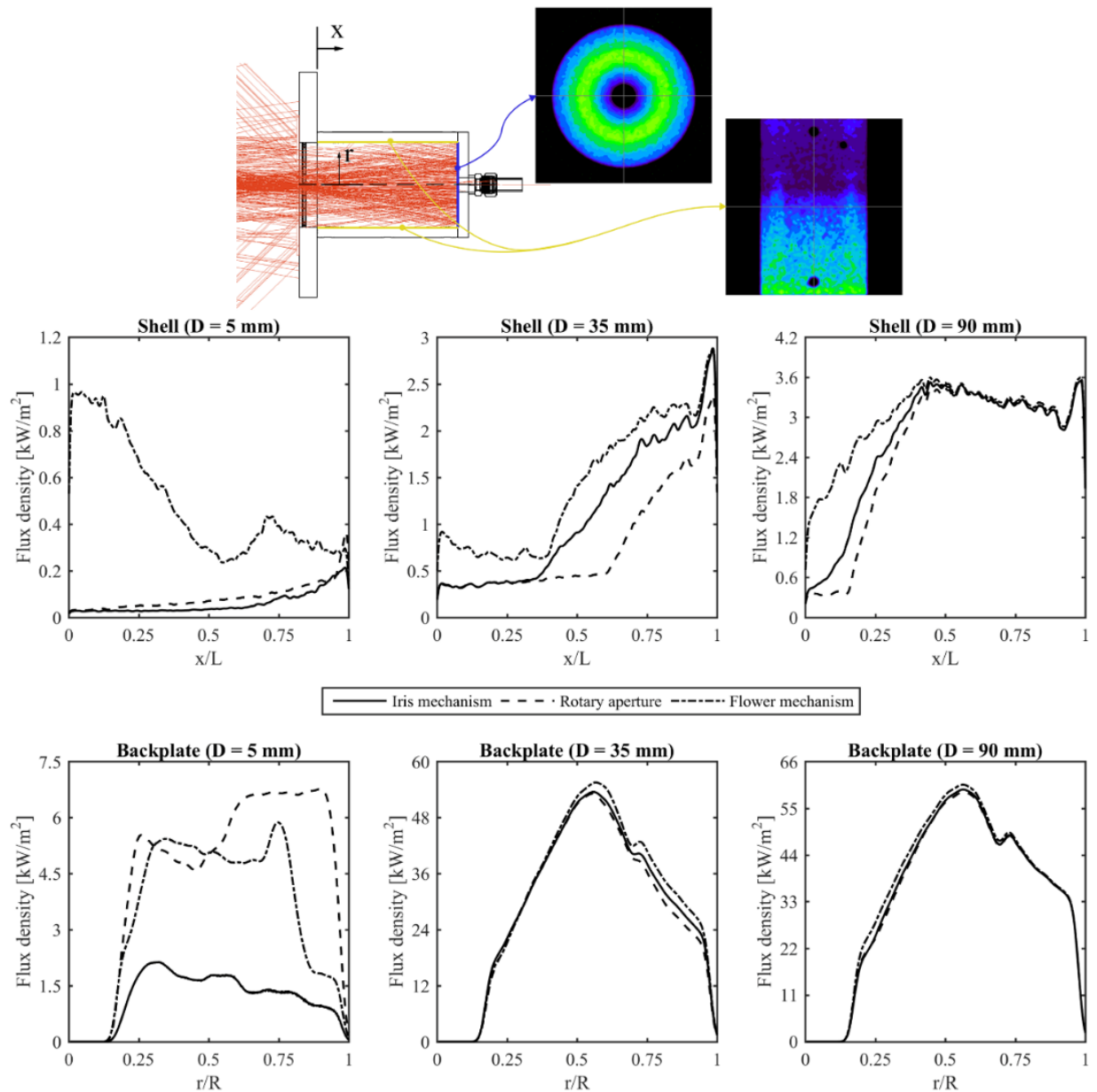

Fig. 7 Flux distribution incident on inner cavity walls in axial (shell) and radial (backplate) direction for three aperture sizes 
In particular, the flower mechanism is able to scatter a big portion of the incoming light bundles when observing the distributions for $\mathrm{D}=5 \mathrm{~mm}$. The radiative power absorbed by the reactor shell is significantly higher in latter case, i.e. $64 \mathrm{~W}$ is absorbed opposed to $3 \mathrm{~W}$ and $11 \mathrm{~W}$ for the iris mechanism and rotary aperture respectively. Increasing the aperture size makes the respective flux distributions for the different apertures come closer to each other. In the case of the flower mechanism, the radiative hotspot gradually shifts from the front of the reactor to the back. Furthermore, the enhanced effect of ray scattering on the irradiation inside the cavity when using the flower aperture has a similar effect to changing the reactor geometry. The latter was demonstrated in Ref. [16] and showed that using a spherical geometry has beneficial effect on the flux distribution. Similarly, the flower mechanism improves the uniformity, however, only at the smaller aperture sizes.

Fig. 8 provides a graphical presentation of the total radiative power and their respective fractions on the cavity walls. The three curves in Fig. 8 depict the change of net radiative power entering the reactor and hitting the stainless steel walls as a function of aperture size. Given the optical and radiative properties of the reactor material, $80 \%$ of this incident bundle is absorbed by the walls. The trends of the curves show that more power reaches the inner cavity when the flower mechanism is employed as variable aperture. For the given aperture range of $\mathrm{D}=0-90 \mathrm{~mm}$, radiative power of up to $863 \mathrm{~W}$ is intercepted by the flower mechanism and reaching the reactor walls. This with respect to $813 \mathrm{~W}$ and $791 \mathrm{~W}$ for the iris mechanism and rotary aperture, respectively, which are 6-8 \% lower. This confirms and extends the results from the enhanced scattering and reflection of the blades, as previously described in Fig. 7. The bars in Fig. 8 clearly reflect the hotspot being shifted from the back of the reactor towards the front, as the aperture enlarges. This mainly applies to the iris mechanism and rotary aperture, where the fraction of power incident on the shell increases from approximately $16 \%$ at $\mathrm{D}=5 \mathrm{~mm}$ to $40 \%$ at $\mathrm{D}=90 \mathrm{~mm}$.

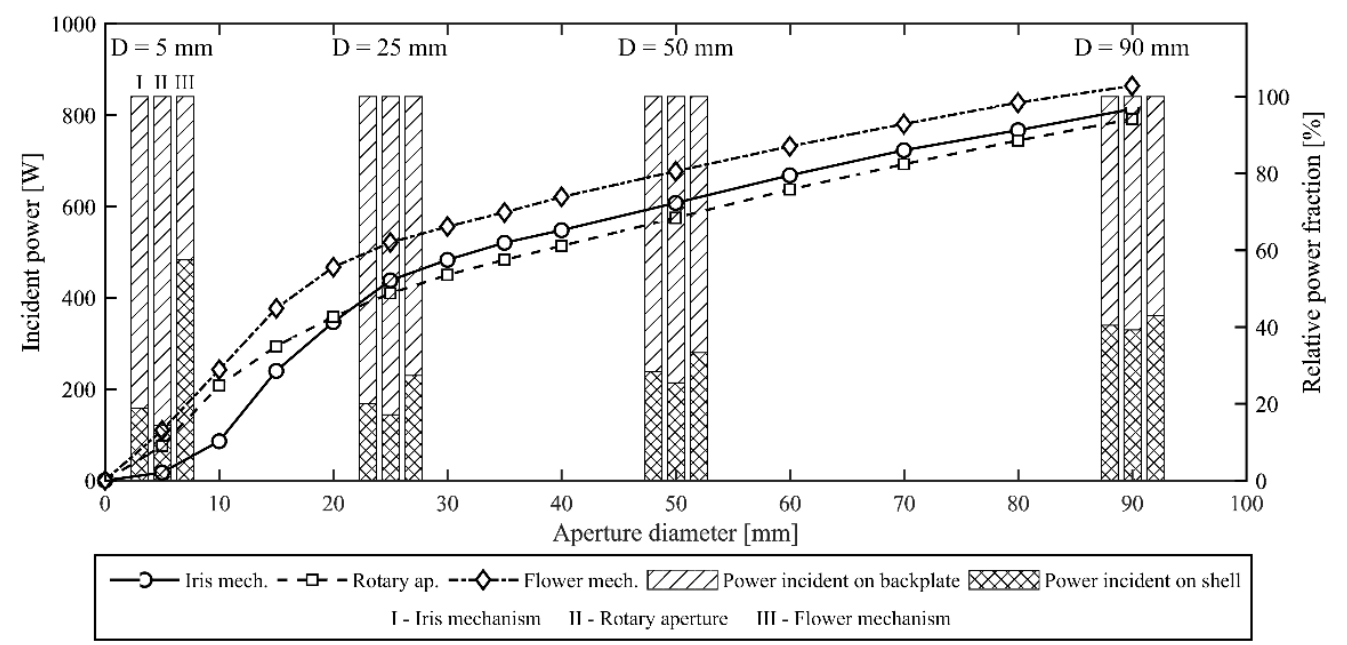

Fig. 8 Radiative power incident on inner cavity walls and the respective fractions on backplate and shell

\section{THERMODYNAMIC ANALYSIS}

This section of the paper briefly discusses the effect of each aperture size on the steady reactor temperature. The thermodynamic model, as was previously elaborated in Ref. 10, showed the significance of the aperture opening factor (AOF). The factor describes the ratio of power intercepted by the aperture to the available power before interception. Analogously, the AOF and resulting Dose-Response gamma model fit was computed for the flower aperture based on the results of the optical simulations. The complete analysis was performed for the 155 A power level of the solar simulator. The lumped parameter model from Ref. 10 was further improved. For example, material properties were considered temperature-dependent, energy balance of the reactor was solved over the three parts of the reactor, i.e. front flange, backplate and shell. 
Fig. 9 depicts the prediction of the reactor temperature in steady state according to the thermodynamic analysis. The given numerical results are compared with experimental data for two of the three mechanisms (i.e. iris mechanism and rotary aperture). An average deviation (AAD) of $15.3 \mathrm{~K}$ and $14.7 \mathrm{~K}$ for the iris mechanism and rotary aperture, respectively, is observed. Thus, temperature prediction of the numerical model is validated and assumed to be accurate for the flower mechanism as well. In Ref. 10, it was observed that regardless of the power level of the simulator, higher reactor temperatures were achieved by implementation of the rotary aperture. This was due to both the error of approximating the iris' crosssectional area as a circle and the reduced re-radiation losses because of the rotary disc being positioned in a plane flush to the reactor flange. The temperature trends in Fig. 9 show a comparable evolution between the iris mechanism and flower mechanism. However, a temperature of $740 \mathrm{~K}$ can be achieved with the flower aperture as opposed to $672 \mathrm{~K}$ with the iris mechanism. The consistently higher reactor temperature, as a result of utilizing the flower aperture, follows from the results and discussion made in Section 4 . The temperature trends demonstrate that temperature change of the reactor gains more sensitivity in the lower aperture range, e.g. $\mathrm{D}=30 \mathrm{~mm}$ and below.

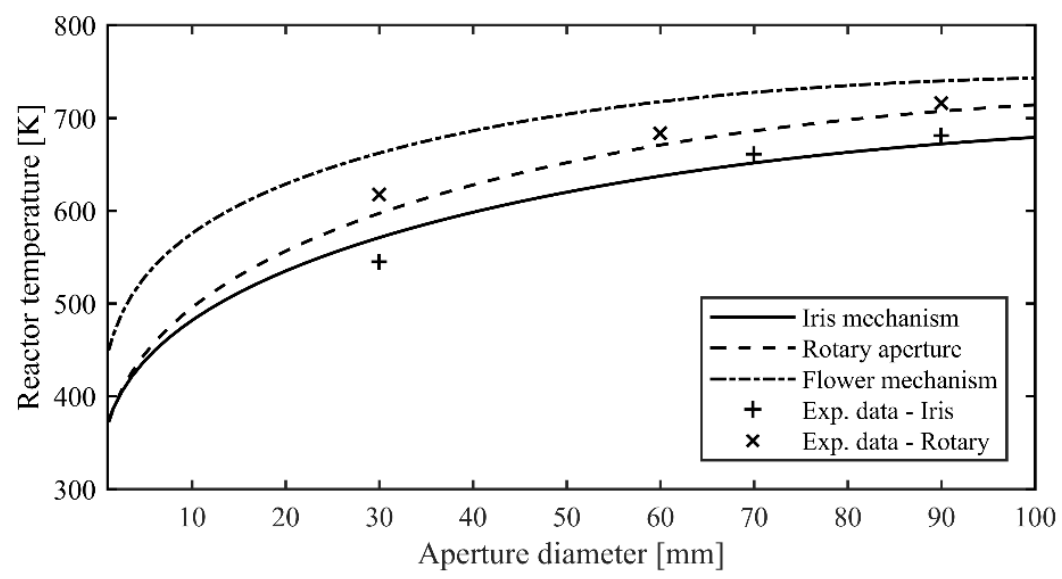

Fig. 9 Steady-state reactor temperature for three variable apertures with experimental data for iris mechanism and rotary aperture

\section{TEMPERATURE CONTROL USING VARIABLE APERTURE MECHANISM}

As it aforementioned, aperture size directly affects solar energy input into the reactor/receiver, and consequently influence the system temperature. This makes the variable aperture concept as a promising option to control the temperature of solar reactors/receivers. Significant effect of aperture size on temperature can be seen in Figure 9, where changing the aperture size from fully closed to fully opened, increased the temperature by around $100 \%$. Numerical simulations conducted in one of our previous studies have also demonstrated the advantage of variable aperture mechanism over gas flowrate adjustment for control of reactor temperature [17]. Figure 10 depicts the closed loop circuit of temperature control using variable aperture mechanism. Based on online temperature measurements from thermocouples on the solar receiver, the error between current temperature and desire setpoint is calculated. Controller uses the error to calculate the control signal, which is then being used by stepper motor to move the blades in variable aperture mechanism to set the desire aperture size. 


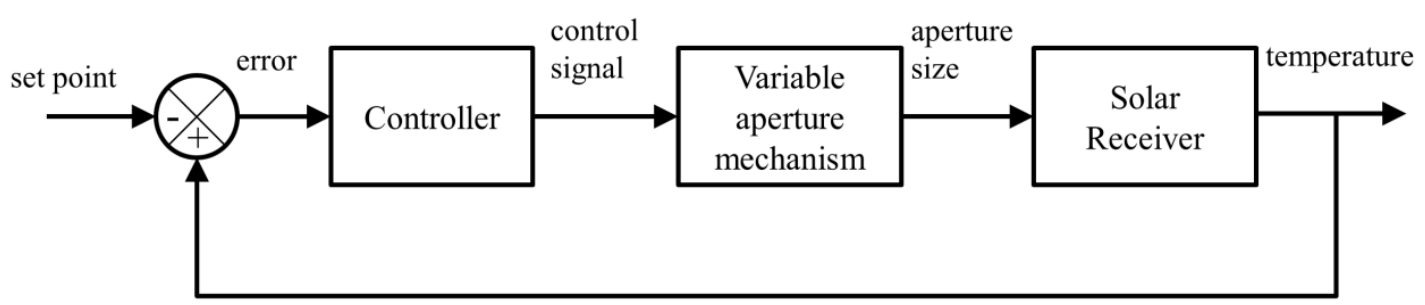

Fig. 10 Closed loop circuit of temperature control using variable aperture mechanism.

As an example, simulation results for temperature control using iris mechanism are presented in Figure $11.7 \mathrm{~kW}$ solar simulator is used as radiative heat source and its power level is changed according to DNI profile of a sunny day at $20^{\text {th }}$ of August, 2016, obtained from NREL database [18]. A numerical model is used to simulate the dynamic temperature variation in the solar receiver. This model was developed by coupling the Monte carlo ray tracing method with energy balances equations of the cavity wall elements as described in reference [19]. Proportional-integral (PI) controller with $k p=0.0033$ and $k_{I}=0.0205$ is used to calculate the control signal to iris mechanism. All the system components were at the ambient temperature before starting the simulation. At early morning, controller opens the aperture completely to allow the temperature to reach to the setpoint as fast as possible. When the temperature reaches the setpoint, controller closes the aperture to keep the temperature constant. Once the setpoint is increased from $600 \mathrm{~K}$ to $680 \mathrm{~K}$ at $\mathrm{t}=9: 00 \mathrm{hr}$, controller opens the aperture to track the new setpoint. At the evening where the DNI decreases, the iris opens again to track the desire setpoint. Reasonable temperature regulation is achieved throughout the day which confirms the performance of the variable aperture concept as a promising option for temperature control.
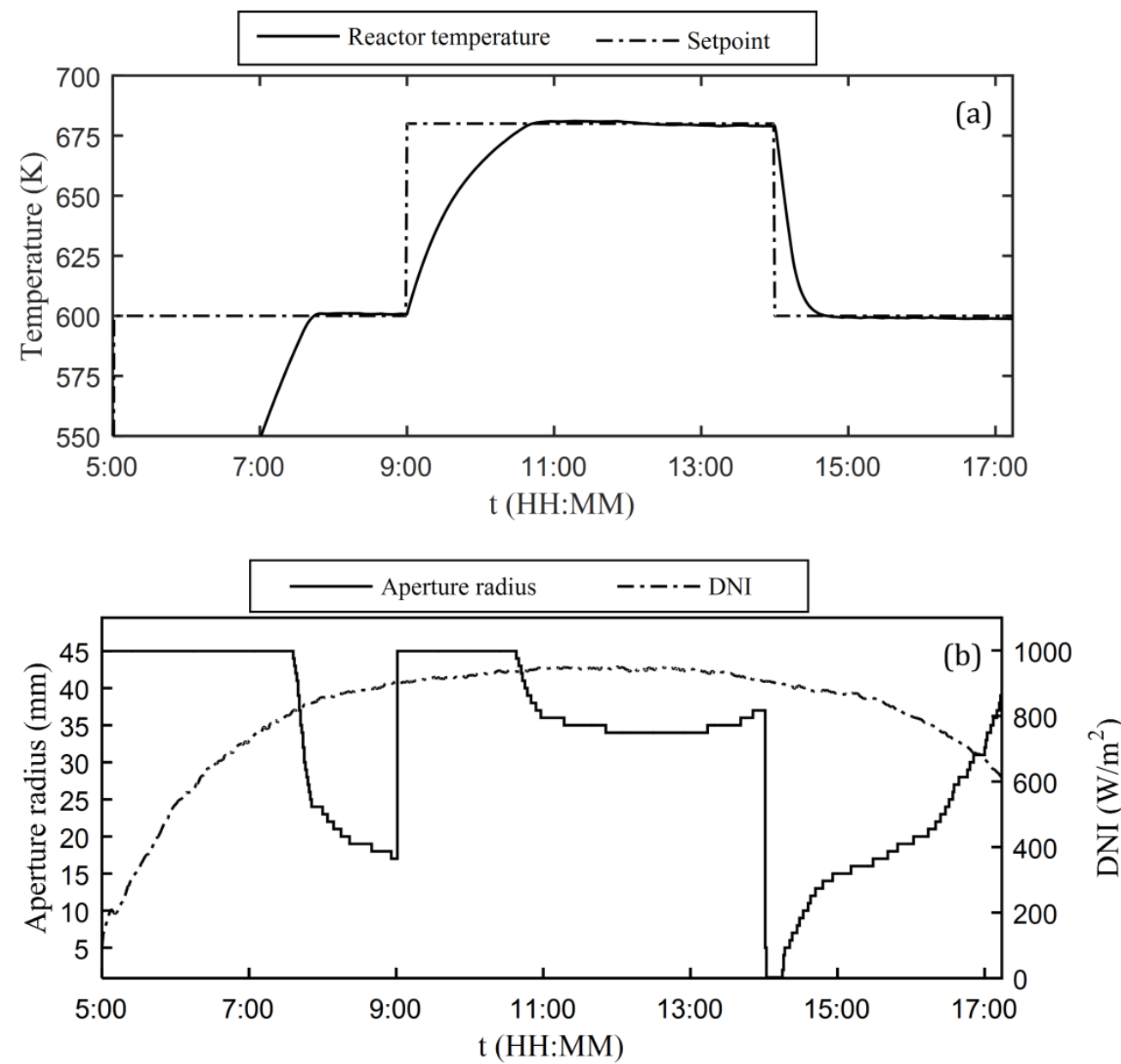

Fig. 11 Simulation results for closed loop temperature control for a sunny day using iris mechanism. (a) Desired setpoint and system output, (b) Control action and DNI profile. 


\section{CONCLUSIONS}

Variable apertures have proven to control the flow of multiple kinds of media. In a similar way to regulating fluid flow in pipes, solar flux which is used in various applications can be adjusted through the adoption of an aperture mechanism. This paper has summarized the extension to the efforts that have been undertaken towards control of light entry inside solar cavity receivers. Three variable aperture mechanisms were compared with each other from a technical, optical and thermodynamic perspective. The effect of the blade geometry on the evolutions of the aperture area was demonstrated for the presented aperture concept. Close approximation of a circular area was shown. Optical simulations were performed in order to assess the impact of the mechanism configuration and blade geometry on the flux distribution incident at the aperture and on the cavity walls, respectively. With consideration of specular properties for the mechanism components, the blades of the flower mechanism showed an enhanced effect on the optical path of the generated light bundles. Results also showed an increased uniformity of flux at small aperture size for the flower mechanism, and thus, controlling this distribution might be a step towards resolving the hotspot problem. The enhanced scattering of the rays manifested itself in an increased incident flux at the walls of $6-8 \%$ higher than the rotary aperture and iris mechanism for the same aperture size. The effect on the flux distribution showed a proportional correlation with the thermal behavior of the reactor cavity. As a result, maximum temperature of $740 \mathrm{~K}$ can be achieved with the flower mechanism. Hence, this comparative study has shown the mutual performance of the variable apertures and the capability to control reactor temperature.

\section{ACKNOWLEDGMENT}

This research has been funded by Impulse Fund project \# IMP/14/049 of KU Leuven.

\section{REFERENCES}

[1] Hakkarainen, E. and Tähtinen, M., "Dynamic Modelling and Simulation of Linear Fresnel Solar Field Model based on Molten Salt Heat Transfer Fluid," AIP Conference Proceedings 1734, 070014, pp. 1-9, (2016).

[2] Tuchelt, M., "Solar Energy Control," U.S. Patent Application No. 11/631,626, (2008).

[3] Ophoff, C. and Ozalp, N., “A Novel Iris Mechanism for Solar Thermal Receivers," Journal of Solar Energy Engineering, 139(6), pp. 1-10, (2017).

[4] Jubb, A., "Solar heat aperture control apparatus," U.S. Patent Application No. 952,215, (1978).

[5] Beeman, R. and Brajkovich, S.J., "Variable orifice using an iris shutter," U.S. Patent Application No. 760,305, (1977).

[6] Noguchi, K., "Iris type light quantity adjusting device, lens device, and image-taking apparatus," U.S. Patent Application No. 10/175,467, (2002).

[7] Bagby, J.P., “Automatic exposure mechanism with magnetic control of iris blades," U.S. Patent Application No. 3,082,674, (1958)

[8] Seo, H.W., Chae, J.B., Hong, S.J., Rhee, K., Hyeon Chang, J., and Chung, S.K., "Electromagnetically Driven Liquid Iris," Sensors and Actuators A: Physical, 231, pp. 52-58, (2015).

[9] Van den Langenbergh, L., Ophoff, C., Ozalp, N., "An iris mechanism driven temperature control of solar thermal reactors," Proceedings of the 1st Thermal and Fluid Engineering Summer Conference, TFESC-13250, pp. 1-15, (2015).

[10] Ophoff, C., Korotunov, S., and Ozalp, N., "Optimization of Design and Process Parameters for Maximized and Stable Solar Receiver Efficiency," Proceedings of the 2nd Thermal and Fluid Engineering Summer Conference, TFEC-IWHT2017-18225, pp. 1-15, (2017).

[11] Sarwar, J., Georgakis G., LaChance, R., and Ozalp N., "Description and characterization of an adjustable flux solar simulator for solar thermal, thermochemical and photovoltaic applications," Solar Energy, 100, pp. 179-194, (2014).

[12] Lee, H., Chai, K., Lee, S., Yoon, H., Yu, C., and Kang, Y., "Optical performance evaluation of a solar furnace by measuring the highly concentrated solar flux," Energy, 66, pp. 63-69, (2014).

[13] Roldan, M.I., and Monterreal, R., "Heat flux and temperature prediction on a volumetric receiver installed in a solar furnace," Applied Energy, 120, pp. 65-74, (2014).

[14] Li, Z., Tang, D., Du, J., and Li, T., "Study on the radiation flux and temperature distributions of the concentrator-receiver system in solar dish/Stirling power facility," Applied Thermal Engineering, 31(10), pp. 1780-1789, (2011).

[15] Lambda Research Corporation, 2017, “TracePro (Version 7.4)," Lambda Research Corporation, Littleton, MA, accessed Sep. 5, 2017, https://www.lambdares.com/tracepro 
[16] Costandy, J., El Ghazal, N., Mohamed, M.T., Menon, A., Shilapuram, V., and Ozalp, N., "Effect of reactor geometry on the temperature distribution of hydrogen producting solar reactors," Int. Journal of Hydrogen Energy, 37(21), pp. 16581-16590, (2012).

[17] Abedini Najafabadi, H., Ozalp, N., "Development of a Control Model to Regulate Temperature in a Solar Receiver" Renewable Energy, 111, pp. 95-104, (2017).

[18] http://www.nrel.gov/midc/srrl\%5Fbms/.

[19] Abedini Najafabadi, H., Ophoff, C., Ozalp, N., "Development of an optimal control strategy to regulate temperature in a solar receiver", Proceedings of the 2nd Thermal and Fluid Engineering Conference, (TFEC2017), Las Vegas, NV, USA, April 2017. 